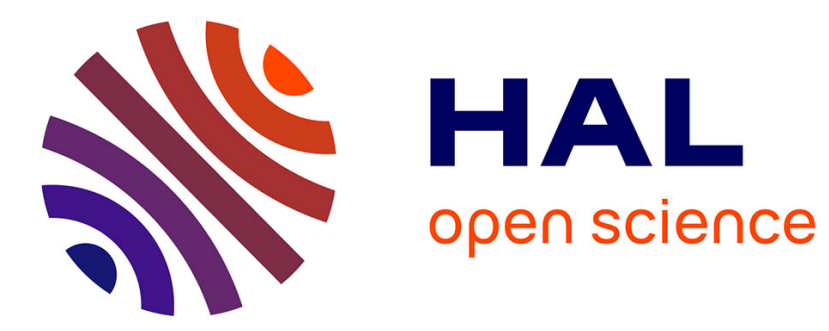

\title{
Preparation of doublet, triangular, and tetrahedral colloidal clusters by controlled emulsification
}

Djamal Zerrouki, Benjamin Rotenberg, Sébastien Abramson, Jean Baudry, Cécile Goubault, Fernando Leal Calderon, David J. Pine, Jérôme Bibette

\section{- To cite this version:}

Djamal Zerrouki, Benjamin Rotenberg, Sébastien Abramson, Jean Baudry, Cécile Goubault, et al.. Preparation of doublet, triangular, and tetrahedral colloidal clusters by controlled emulsification. Langmuir, 2006, 22, pp.57. 10.1021/la051765t . hal-00079315

\section{HAL Id: hal-00079315 https://hal.science/hal-00079315}

Submitted on 6 Dec 2018

HAL is a multi-disciplinary open access archive for the deposit and dissemination of scientific research documents, whether they are published or not. The documents may come from teaching and research institutions in France or abroad, or from public or private research centers.
L'archive ouverte pluridisciplinaire HAL, est destinée au dépôt et à la diffusion de documents scientifiques de niveau recherche, publiés ou non, émanant des établissements d'enseignement et de recherche français ou étrangers, des laboratoires publics ou privés. 


\section{Preparation of doublet, triangular, and tetrahedral}

\section{colloidal clusters by controlled emulsification}

Djamal Zerrouki ${ }^{l}$, Benjamin Rotenberg $^{l+}$, Sébastien Abramson ${ }^{l *}$, Jean Baudry $^{l}$, Cécile Goubault ${ }^{l}$, Fernando L. Calderon ${ }^{2}$, Dave J. Pine ${ }^{3}$ and Jérome Bibette ${ }^{l}$

${ }^{1}=$ Laboratoire des Colloïdes et Matériaux Divisés - UMR 7612 (UPMC-CNRS-ESPCI) ESPCI, 10 rue Vauquelin, 75231 Paris cedex 05 (France) ${ }^{2}=$ Laboratoire des Milieux Dispersés Alimentaires, Institut des Sciences et Techniques des Aliments de Bordeaux, Av. des facultés, 33405, Talence cedex, (France) ${ }^{3}=$ Department of Physics, New York University, 4 Washington Place, New York, NY 10003 (USA)

${ }^{+}=$present adress : Laboratoire des Liquides Ioniques et Interfaces Chargées (UMR 7612) Université P. \& M. Curie, 4, place Jussieu, 75252 Paris Cedex 05 - (France)

*=Corresponding author. Tel : 33140795234 ; fax: 33140795245 ; e-mail address: sebastien.abramson@espci.fr 


\section{Abstract}

We describe a six-step method for making colloidal clusters of 2,3 or 4 silica particles with a radius of $1.2 \mu \mathrm{m}$. This method, originally described by Manoharan et al. (V. N. Manoharan, M. T. Elsesser, D. J. Pine, Science, 2003, 301,483), is based on the encapsulation of silica spheres in emulsion droplets. The originality of our work lies in the preparation of monodisperse emulsions, which allows us to obtain some high yields of small aggregates over a wide range of conditions. Using optical microscopy and disc centrifugation, we show that the relative fractions of 2,3 and 4 particle aggregates is controlled by the emulsification conditions, particularly the concentration of silica in the dispersed phase. Our best yields are obtained using low to moderate shear rates, a highly viscous continuous phase, and intermediate amounts of silica. The sedimentation of the colloidal solution into a gradient of concentration leads to aqueous suspensions of identical clusters. Since the overall process can easily be scaled up, large quantities of identical clusters may be prepared, which should allow the thermodynamic properties of these new colloidal objects to be measured for the first time. These non-spherical particles could serve as building blocks for more complex assemblies, such as colloidal crystals which could find applications as photonic materials. 


\section{Introduction}

Colloidal crystals can be viewed as the mesoscopic counterpart of atomic or molecular crystals. They are comprised of particles with dimensions from a few nanometers to about one micrometer that order into $2 \mathrm{D}$ or $3 \mathrm{D}$ periodic lattices. They have been used to explore diverse phenomena such as crystal growth $^{1-3}$ and the glass transition. ${ }^{4}$ and have many interesting applications for sensors, ${ }^{5}$ in catalysis, ${ }^{6}$ advanced coatings, ${ }^{7}$ and for optical/electro-optical devices for information processing and storage. ${ }^{8,9}$ In particular, their unusual optical properties, namely the diffraction of visible light and the existence of a photonic stop band, make them ideal candidates for the development of photonic materials. ${ }^{8,10-13}$ They may lead to the fabrication of more efficient light sources, miniature waveguides, detectors, modulators, and circuits.

Any study of colloidal crystals requires the preparation of monodisperse colloidal particles that are uniform in size, shape, composition and surface properties. Monodisperse spherical colloids of various sizes, composition, and surface properties have been prepared by numerous synthetic strategies. ${ }^{14}$ However, the direct preparation of crystal phases from spherical particles usually leads to a rather limited set of close-packed structures (hexagonal close packed, face-centered cubic, or body-centered cubic structures).

Relatively few studies exist on the preparation of monodisperse non-spherical colloids. In general, direct synthetic methods are restricted to particles with simple shapes like rods, spheroids or plates. ${ }^{15}$ An alternative route for the preparation of uniform particles with a more complex structure might consist in the formation of discrete uniform aggregates of self-organized spherical particles. The use of colloidal clusters with a given number of particles, with controlled shape and dimension, could lead to colloidal crystals with unusual symmetries. ${ }^{16}$ 
The preparation of colloidal clusters with well-defined structures is difficult because the interactions between the colloids are generally non-directional. Therefore, when suspended in a liquid medium, spherical colloids tend to form discrete aggregates but with a very broad size distribution and no welldefined shape. An approach that has proved to be efficient consists of physically confining the particles using a template. ${ }^{17-26}$ This allows the clusters to be isolated from each other by the walls of the templates so that uncontrolled aggregation between the clusters is prevented. The structure of the cluster is essentially controlled by the respective shapes and dimensions of the template and of the particles. ${ }^{20}$

Different sorts of templates can be used to direct the self assembly of spherical colloids into well-defined clusters. Some success has been achieved by patterning surfaces with chemical functions ${ }^{17,18}$ or with geometric structures. ${ }^{19,20}$ Aggregates with well defined shapes like polygonal or polyhedral clusters, rings, and chains were obtained. Other authors relate the use of emulsion droplets as template by adsorbing particles on their surface ${ }^{21,22}$ or by confining them into their core..$^{23,24}$ However the majority of these works led to aggregates with a large number of particles.

Recently Manoharan et al. reported a method for fabricating small clusters of particles using emulsions. ${ }^{25,26}$ Microspheres (diameter $844-900-1170 \mathrm{~nm}$ made from polystyrene, silica or PMMA) were dispersed in an oil-in-water emulsion with each oil droplet containing a small number $N$ of spheres. The oil was then preferentially evaporated. Removing the liquid from a droplet containing particles bound on its surface generated compressive forces that drew the particles together into compact clusters. Surprisingly, for all $N<11$, the final particle packings were unique: the observed packings closely corresponded to those previously identified as minimizing the second moment of the mass distribution, $\mathbf{M}=\sum_{\mathbf{i}}\left(\mathbf{r}_{i}-\mathbf{r}_{\mathbf{0}}\right)^{2}$, where $\mathbf{r}_{0}$ is the center of mass of the cluster. Separation of the aggregates by density gradient centrifugation resulted in aqueous suspensions containing relatively high amounts of identical stable clusters $\left(10^{8}\right.$ to $10^{10}$ clusters). However, lack of control of the emulsification conditions 
led to polydisperse emulsions and severely limited the yield of each type of cluster. Indeed, encapsulation of particles in polydisperse emulsions led to a wide variation in the number of spheres in different droplets. Consequently, in order to obtain a relatively high proportion of small aggregates $(N<11)$, they used a small volume fraction of colloids in the oil phase.

In this communication, we show that the preparation of monodisperse droplets by controlled emulsification is highly effective for making large quantities of small clusters. In this case, the mean number of colloid per droplet can be easily adjusted. More importantly, the statistical distribution of the number of spheres per droplet is narrowed significantly by tightly controlling the droplet diameter and by narrowing its distribution. This leads to an increase of the proportion of small aggregates over a large range of conditions. More precisely, we show that controlling of the amount of colloid in the dispersed phase, the viscosity of the continuous phase, and the shear rate during emulsification are all important for obtaining a high yield of clusters of a given size. We focus on the preparation of clusters containing fewer than five particles, i.e. doublet, triangular and tetrahedral clusters. Controlled assembly of these kind of aggregates may lead to interesting colloidal crystals particularly with a diamond-like structure. ${ }^{16}$

\section{Experimental Section}

Synthesis of hydrophobic monodisperse silica spheres. Hydrophilic silica spheres were synthesized by hydrolysis of tetraethylorthosilicate (TEOS 98\%, ACROS) in a water medium containing ethanol and ammonia using a modified seeded growth procedure originally described by Stöber and Fink. ${ }^{27,28}$ To prepare the seeds, $50 \mathrm{~mL}$ of TEOS was added to $100 \mathrm{~mL}$ of a mixture of water/ethanol/ammonia $\left(78 / 11 / 11 \%\right.$ in volume) and the resulting solution was stirred for 10 min at $40^{\circ} \mathrm{C}$. To make the final particles, we added $25 \mathrm{~mL}$ of TEOS and $50 \mathrm{~mL}$ of water/ethanol/ammonia solution (79/16/5\% in volume) every 10 minutes to the original suspension of seed particles at $40^{\circ} \mathrm{C}$. This procedure was repeated until the desired diameter was reached. The shape, mean radius, and polydispersity of the final 
particles $(1.21 \mu \mathrm{m} \pm 0.08)$ were determined using dynamic light scattering (DLS) and transmission electron microscopy (TEM). To make the silica particle surfaces hydrophobic, we used a method based on a silanization reaction of the hydrophilic silica surface with octyldimethylchlorosilane (ODMCS, $95 \%$, Aldrich). ${ }^{29} 5 \mathrm{~mL}$ of ODMCS diluted in 30ml DMF was added to $10 \mathrm{~g}$ of hydrophilic silica dispersed in propylene carbonate. This mixture was incubated at $75^{\circ} \mathrm{C}$ under $\mathrm{N}_{2}$ atmosphere with a gentle stirring for $24 \mathrm{~h}$. The resulting suspension was rinsed using DMF and acetone to remove ODMCS, redispersed and further rinsed twice with octane.

Emulsion preparation and shearing. The silica particles were dispersed in octane with a mass fraction $\phi_{i n t}$. The suspension was sonicated and vortexed. To fabricate the premix oil-in-water emulsion, this mixture was progressively incorporated to an aqueous solution containing $1 \% \mathrm{w} / \mathrm{w}$ of Synperonic PE/F68 (Uniquema) and 15-30\% w/w of polyvinylpyrolidone (PVP-K90, Aldrich). The concentration of dispersed phase (silica/octane) was $30 \% \mathrm{w} / \mathrm{w}$. The Synperonic PE/F68 surfactant that was added to stabilize the $\mathrm{O} / \mathrm{W}$ emulsion is a triblock copolymer of ethylene oxide(EO) and propylene oxide $(\mathrm{PO})$ with an average formula of $75 \mathrm{EO} / 30 \mathrm{PO} / 75 \mathrm{EO}$, an average molecular weight of $\mathrm{M}_{\mathrm{W}} \sim 8400 \mathrm{~g} / \mathrm{mol}$ and a hydrophilic to lipophilic balance of $\mathrm{HLB}=29$. To increase the viscosity of external water phase, we added PVP-K90 which is a hydrophilic polymer with a molecular weight of $\mathrm{M}_{\mathrm{w}} \sim 360,000 \mathrm{~g} / \mathrm{mol}$. To obtain a quasi monodisperse emulsion, we used a Couette apparatus ${ }^{30-33}$ (manufactured by TSR Co. ${ }^{34}$ ). The premix emulsion (minimal amount of $15 \mathrm{~g}$ ) was injected with a velocity of $V_{i n j}=0.7 \mu \mathrm{m} / \mathrm{s}$ and sheared between two concentric cylinders, the inner cylinder rotating at a selected angular velocity $\left(0\right.$ to $\left.71.2 \mathrm{rad} \mathrm{s}^{-1}\right)$. The corresponding shear rate $\left(0\right.$ to $\left.14200 \mathrm{~s}^{-1}\right)$ is given by the equation $\gamma \approx \mathrm{R} \omega /\left(\mathrm{R}_{\mathrm{i}}-\mathrm{R}\right)$ where $\omega, R$ and $R_{i}$ are the angular velocity, the radius of the inner cylinder $(19.89 \mathrm{~mm})$ and the radius of the outer cylinder $(19.99 \mathrm{~mm})$, respectively. 
Cluster preparation and separation. The sheared emulsion was diluted in a $1 \% \mathrm{w} / \mathrm{w}$ aqueous solution of Synperonic PE/F68 with a mass fraction of 10\%. The crude silica cluster suspension was obtained by removing the octane by distillation at $125^{\circ} \mathrm{C}$ under atmospheric pressure during $60 \mathrm{~min}$ under continuous gentle stirring. At the same time, the suspension was concentrated by evaporation of the appropriate amount of water to reach a concentration of $2 \% \mathrm{w} / \mathrm{w}$ of silica in water. Clusters were separated by size, using a simple sedimentation process. $50 \mathrm{~mL}$ of the cluster suspension was carefully loaded on a top of a column (height $=400 \mathrm{~mm})$ containing $5 \mathrm{~L}$ of a linear gradient $(3-12 \% \mathrm{w} / \mathrm{w}$ in water) of Ficoll 400 (Aldrich). After 12 hours of sedimentation, the individual bands corresponding to aqueous suspensions of purely identical clusters were extracted with a syringe. The fractions were purified by exchanging several times the aqueous solutions with a $1 \%$ solution of Synperonic PE/F68 in water. In this medium, the clusters were stable during some days.

Characterization methods. The emulsion and cluster suspensions were examined with an optical microscope (Nikon) equipped with an oil immersion objective $(\times 100)$. Clusters in the dried state were imaged with a scanning electron microscope S440 (LEICA) in secondary electron mode after sputter coating with gold.

The cluster size distribution in the crude suspension was determined by sedimentation in a disc centrifuge (model DC 18000, CPS instruments). The disc was first allowed to reach the desired rotating rate/speed $(2300 \mathrm{rpm})$ as a function of the silica density $(\mathrm{d}=2,1)$. The sucrose gradient was formed by injecting $1.6 \mathrm{ml}$ of water with sucrose concentrations of $24,22,20,18,16,14,12,10$ and $8 \% \mathrm{w} / \mathrm{w}$. The sucrose gradient was allowed to run for at least $30 \mathrm{~min}$. Then $0.1 \mathrm{ml}$ of cluster suspension in water was injected in the center of the rotating disc.

The sedimentation time for each cluster type is different, increasing monotonically with the hydrodynamic diameter. Indeed, the sedimentation rate of an aggregate of $\mathrm{N}$ spherical particles in a disc centrifuge can be calculated by equation (1) 


$$
\mathrm{V}=\left(\mathrm{N} \cdot \mathrm{D}_{0}{ }^{3} \cdot \Delta \rho \cdot \omega^{2} \cdot \mathrm{R}\right) /\left(18 \cdot \eta \cdot \mathrm{D}_{\mathrm{h}}\right)
$$

where $\mathrm{D}_{0}$ is the particle diameter, $\Delta \varrho$ is the difference between the densities of the aggregate and the liquid, $\omega$ is the angular frequency of the disc, $R$ is the radius of the disc, $\eta$ is the viscosity of the liquid and $\mathrm{D}_{\mathrm{h}}$ is the hydrodynamic diameter of the aggregate.

For a small $\mathrm{N}$, the hydrodynamic diameter of an aggregate is given by the empirical equation (2) ${ }^{35}$

$$
\mathrm{D}_{\mathrm{h}}=\left(0.518+0.418 \cdot \mathrm{N}^{1 / 2}\right) \cdot \mathrm{D}_{0}
$$

The rate of an aggregate is then related to $\mathrm{N}$ by equation (3) and thus $\mathrm{V}$ is increased with the particle number $\mathrm{N}$

$$
\mathrm{V} \sim \mathrm{N} /\left(0.518+0.418 \cdot \mathrm{N}^{1 / 2}\right)
$$

The concentration of each type of aggregate is determined by continuously measuring the turbidity of the fluid near the outer edge of the rotating disc. The turbidity measurements were converted to a weight distribution using Mie theory light scattering calculations. The scattering cross sections $\mathrm{C}_{\text {scat, } \mathrm{N}}$ were calculated for each type of aggregate relatively to the scattering cross section of a single particle $\mathrm{C}_{\text {scat }, 1}$. The calculated values were 2.05 for doublets, 3.15 for triplets (triangular) and 4.30 for quadruplets (tetrahedral) with a sphere diameter of $1.2 \mu \mathrm{m}$ and an optical index of 1.45 at $470 \mathrm{~nm} .{ }^{36}$ A typical result of this analysis is shown in figure 3. From left to right, the four peaks correspond to single particles, doublets, triplets, and quadruplets, respectively. We have verified the position of each peak is correct using equations (1)-(3)

\section{Results}

Our process for the preparation of aqueous phases of identical clusters was performed in six steps. First, colloidal particles of silica were synthesized. Next, the surface of the particles was made hydrophobic by chemical grafting. Then, an oil-in-water premix emulsion was made by adding an octane suspension of the colloids in an aqueous solution. Controlled shear of the premix was subsequently performed in order to obtain a quasi-monodiperse emulsion. Elimination of the octane 
from the emulsion led to an aqueous suspension of the colloidal clusters. Finally, the clusters were separated by sedimentation in a liquid density gradient column.

Spherical particles of silica were prepared using a method derived from the original Stöber process. ${ }^{27}$ As shown by DLS, they were highly monodisperse with a mean diameter of $d=1.2 \mu \mathrm{m}$. By grafting the silane agent octyldimethylchlorosilane (ODMCS) onto their surface, ${ }^{29}$ the particles could be easily suspended in organic media such as octane. The mass fraction of hydrophobized silica in octane $\left(\phi_{\text {int }}\right)$ was varied from $1 \%$ to $40 \%$. The premix emulsion was made by adding the organic colloidal solution in an aqueous phase containing a hydrophilic surfactant (Synperonic PE/F68) and a thickening agent (PVP-K90). ${ }^{30}$ PVP was added in order to increase the viscosity ratio between the continuous phase and the dispersed phase and thereby improve droplet breakup of this crude emulsion during the subsequent shearing step. The amount of PVP in the aqueous phase was varied for studying the influence of the viscosity of the continuous phase on the shearing process. Figure 1 shows an optical micrograph of a premix emulsion $\left(\phi_{i n t}=17 \%\right)$. The premix is highly polydisperse (sizes range from 2 to more than 20 $\mu \mathrm{m})$ and the droplets generally contain a large number of silica particles.

The premix emulsion was sheared in a precision Couette cell..$^{31,32}$ This method is capable of making large quantities of quasi-monodisperse emulsions with a great reproducibility. Water-in-oil, oil-in-water, water-in-oil-in-water monodisperse emulsions with various compositions have been prepared previously using this method. ${ }^{30-33}$ To control the mean diameter and the polydispersity of the droplets, we varied the shear rate from 1458 to $8331 \mathrm{~s}^{-1}$. Figure 2 shows an optical micrograph of the emulsion after shearing at $3124 \mathrm{~s}^{-1}$. Compared to the premix, the droplets are less polydisperse with a much smaller size and generally contain only a small number of particles. Thus, as previously observed for simple oil-in-water emulsions, ${ }^{31-33}$ the controlled shear allowed a regular fragmentation of the premix droplets. 


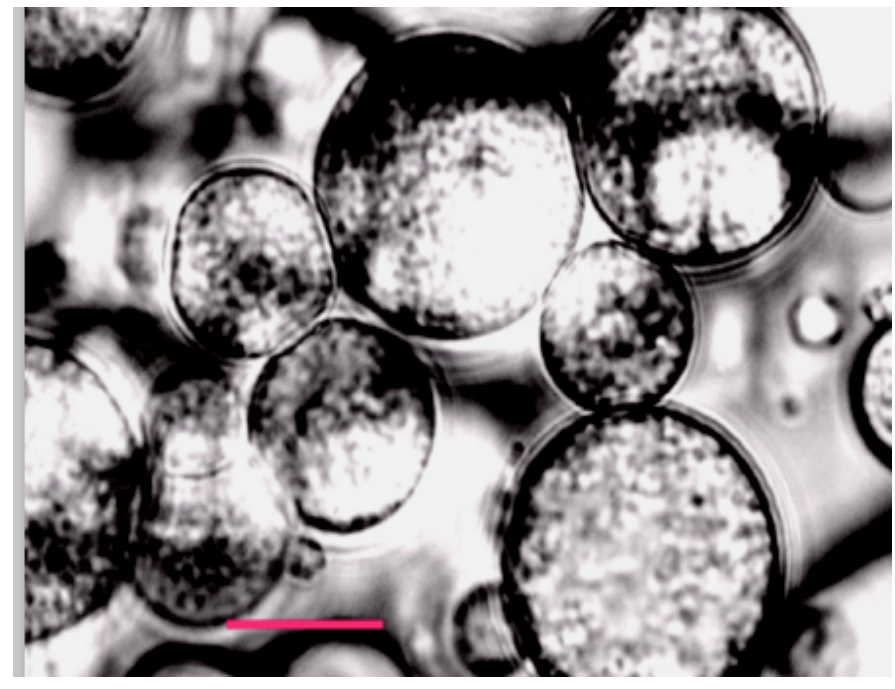

Figure 1 : Premix emulsion

(scale bar 10 $\mu \mathrm{m}$ )

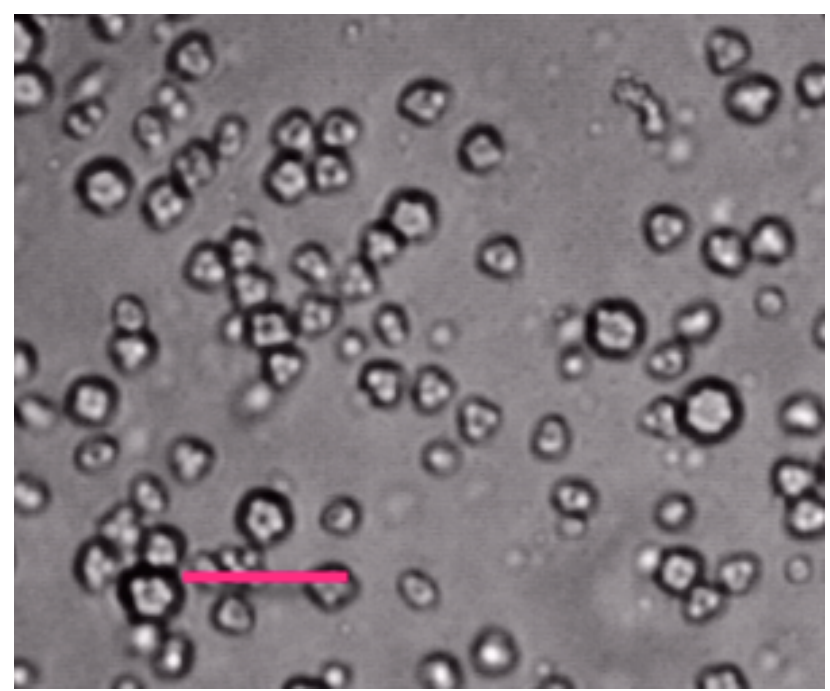

Figure 2 : Emulsion after shearing at $3124 \mathrm{~s}^{-1}$ (scale bar $10 \mu \mathrm{m}$ )

The sheared emulsion was diluted in a solution of Synperonic PE/F68 in water. When octane was removed by slow evaporation, aggregation of the particles in the emulsion droplets occurred and thus a stable aqueous suspension of colloidal clusters was formed. As already observed by Manahoravan et al. and Yi et al., ${ }^{25,26}$ removal of the surfactant and the thickening agent from the colloidal suspension by exchanging with pure water led to aggregation of the clusters. Therefore, adsorption of the Synperonic surfactant onto the clusters to prevent their aggregation is a crucial point.

The proportion of each type of cluster as a function of their particle number $N$ in the colloidal suspension was determined by disc centrifugation. The sedimentation rate of the clusters, which move under the influence of centrifugal force, increases with particle number, as shown in equations (1)-(3) Light scattering of the separated clusters was used to determine the presence of aggregates of different particle number in the original suspension. Figure 3 shows an example of the cluster size distribution in a suspension, each peak corresponding to a cluster with a given number of particles. 


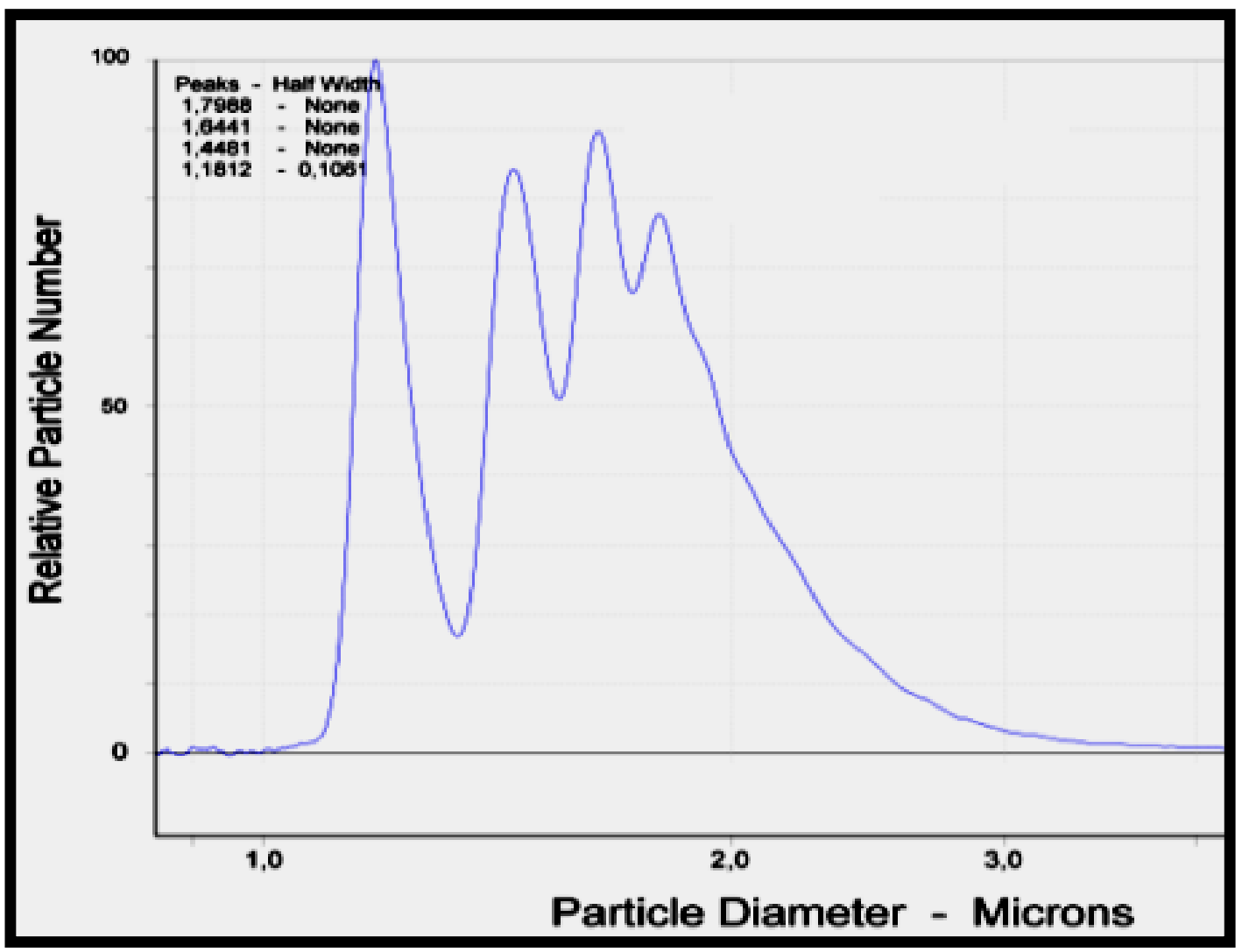

Figure 3 : Disc centrifuge characterization $\left(\%(P V P)=30 \%, \phi_{\text {int }}=17 \%\right.$, shear rate $\left.3124 \mathrm{~s}^{-1}\right)$.

From left to right, the four well-defined peaks correspond respectively to single particles, doublets, triplets and quadruplets.

The highest proportion of aggregates of $N=2$ particles (doublets) in the colloidal suspension was obtained by shearing at $4165 \mathrm{~s}^{-1}$ a premix emulsion containing 30\% of PVP in the continuous phase with a mass fraction $\phi_{\text {int }}=5 \%$ of hydrophobic silica in the dispersed phase. After evaporation of octane, disc centrifugation showed that the suspension contains $21 \%$ of doublets. The highest proportions of $N=3$ (triplets) and $N=4$ (quadruplets) clusters (17\% and 13\%, respectively) were found for $30 \%$ of PVP at a shear rate of $3124 \mathrm{~s}^{-1}$ and with mass fractions $\phi_{\mathrm{int}}=10 \%$ and $17 \%$ of silica for triplets and quadruplets, respectively.

We separated the clusters on the basis of their sedimentation velocity by introducing the crude colloidal suspension into a column containing a concentration gradient of Ficoll. Indeed, as pointed out 
earlier, the sedimentation rate of the clusters depends on the number of particles. Thus, after 12 hours, we observed that the original colloidal suspension was separated in five well defined bands. The first band consisted of single particles; the next three bands consisted of doublets, triplets, and quadruplets, respectively. The last much larger band was made up of clusters containing $N \geq 5$ particles. Using this method, we can obtain aqueous suspensions containing identical clusters of $N=2,3$ or 4 particles. The maximum yield with respect to the initial amount of silica in the crude suspension is about $13 \%$ for doublets, $15 \%$ for triplets and $14 \%$ for quadruplets. These optimal yields concern different experiments. At this stage, working with a crude cluster suspension containing about $1 \mathrm{~g}$ of silica, we obtained about one hundred milligrams for each type of cluster $\left(10^{10}\right.$ to $10^{11}$ in number $)$, which is more than was obtained by Manoharan et al. who reported obtaining $10^{8}$ to $10^{10}$ identical clusters. ${ }^{25}$ However, since the sedimentation step can certainly be scaled up to several grams of silica, the process could be used to prepare about one gram of identical clusters.

Figure 4 shows the scanning electron micrograph of the pure phases of colloidal clusters after depositing them on a silicon wafer and removing the water. As observed by Manoharan $e t$ al. ${ }^{25}$ and by Yi et al., ${ }^{26}$ the triplets and the quadruplets adopt triangular and the tetrahedral configurations, respectively.
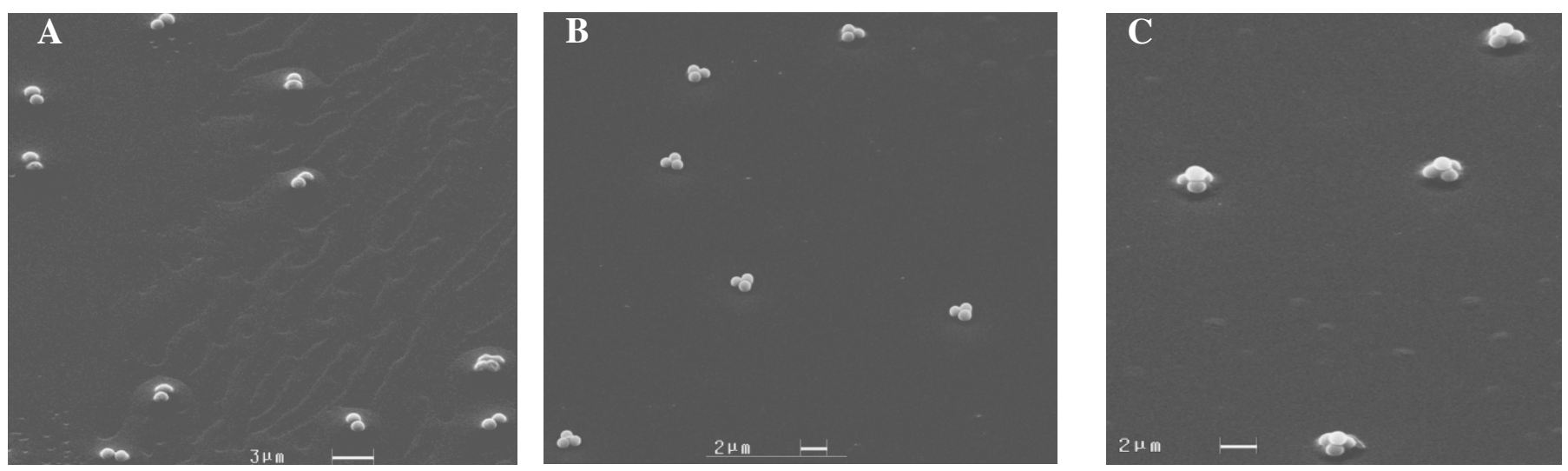

Figure 4: Scanning electron micrographs of the silica colloidal clusters 


\section{Discussion}

From these results, we see that careful control of the parameters during emulsification can increase the percentage of small aggregates in the cluster suspension and can lead to large quantities of identical clusters $(N=2,3,4)$ after the separation step. The composition of the cluster suspension is notably influenced by three parameters: the percentage of silica, the viscosity of the continuous phase, and the shear rate.

We first comment about the role of the silica amount. Indeed, the composition of the cluster suspension is controlled principally by the concentration of silica, which we varied from $1 \%$ and $40 \%$ $\mathrm{w} / \mathrm{w}$ in the oil (octane) phase. We find that the fraction of aggregates containing more than 4 particles never exceeds $35 \%$ so long as the continuous phase is sufficiently viscous (30\% of PVP in the continuous phase). The fraction of singlets, doublets, triplets, or quadruplets can be favored by varying the concentration of silica, as shown in figures 5-8. At very low concentrations of silica $\left(\phi_{\text {int }}=1 \%\right)$, single particles dominate. For concentrations of silica between $5 \%$ and $25 \%$, fragmentation of the emulsion leads to a majority of small aggregates. Doublets are favored for $\phi_{\text {int }}$ between $1 \%$ and $5 \%$, triplets are favored for $\phi_{\text {int }}$ between $5 \%$ and 10\%, and quadruplets are favored for $\phi_{\text {int }}$ between $10 \%$ and $17 \%$, independently of the shear rate used. Thus, increasing the concentration of silica from $1 \%$ to $17 \%$ favors aggregates of increasing number of particles.

Mason and Bibette observed that the droplet radius of oil-in-water emulsions increases when the viscosity ratio between the continuous and dispersed phases is increased, since droplets having too high internal viscosity resist to fragmentation. ${ }^{31}$ Here, the addition of increasing amounts of hydrophobized silica in octane leads to an increase of the viscosity of the droplet phase. Thus, for a same shear rate, 
fragmentation will be stronger with a small amount of silica so that smaller droplets containing fewer particles will be obtained. Curiously, when the amount of silica is greater than $17 \%$, the proportion of single particles once again dominates. In this case, optical microscopy reveals that in the premix emulsion some silica spheres adsorb on the water-oil interface (above $30 \%$ of silica in octane, many particles strongly adsorb on the outer surface of the oil droplets, which tend to be deformed). This modifies the viscoelastic properties of the oil droplet interface. This, in turn, may significantly change the fragmentation mechanism of the oil droplets. In fragmentation of a conventional emulsion, the droplets are first elongated in response to the viscous stress, as early described by Taylor. ${ }^{37}$ Grace ${ }^{38}$ and more recently, Mabille et al.. ${ }^{33}$, have shown that for an external to internal viscosity ratio larger than 0.5 , the deformed droplets undergo capillary instabilities and hence, fragment into small monodisperse droplets. Here, the octane phase may be irregularly fragmented by expulsion of small droplets containing single particles.

To study the effect of the viscosity of the continuous phase, we prepared two sets of premix emulsions $\left(\phi_{i n t}=5-30 \%\right.$ of silica), one containing $15 \%$ and the other $30 \%$ of PVP in the aqueous phase. Whatever the shear rate, the fraction of aggregates of $N \geq 5$ particles was much smaller for the emulsions containing the higher concentration of PVP. For example, at a shear rate of $3124 \mathrm{~s}^{-1}$ with $30 \%$ of silica in octane, the proportion of large aggregates is $60 \%$ with $15 \%$ of PVP, but drops to $35 \%$ with $30 \%$ of PVP. Our results can easily be interpreted using the results of the study of Goubault et al. on double water-in-oil-in-water emulsions. ${ }^{30}$ They observed that when the concentration of thickening agent in the aqueous phase is increased, smaller oil droplets with a narrower size distribution are obtained. This trend was explained by an increase in the viscosity of the continuous phase leading to a higher viscous stress during the shear and to a better fragmentation of the oil droplets. ${ }^{39}$ In simple oil-in-water emulsions, P Aronson had already shown that the increase in viscosity of the continous phase leads to smaller oil droplets. ${ }^{40}$ In our case, the viscosity of the continuous phase should also be high (this is 
obtained by adding a large amount of PVP) in order to achieve effective fragmentation of the premix oil droplets that initially contain a high concentration of particles. This leads to a high percentage of small aggregates.

The effect of the shear rate was studied for the different amounts of silica in octane. In all cases, the highest proportions of aggregates containing a small number of particles are obtained for a low to moderate shear rate $\left(\gamma=1458 \mathrm{~s}^{-1}\right.$ to $\left.4165 \mathrm{~s}^{-1}\right)$. At elevated shear rates $\left(\gamma=8831 \mathrm{~s}^{-1}\right)$, single particles are favored because the high viscous stress leads to fragmentation of the premix into droplets of small radius that contain only one particle.

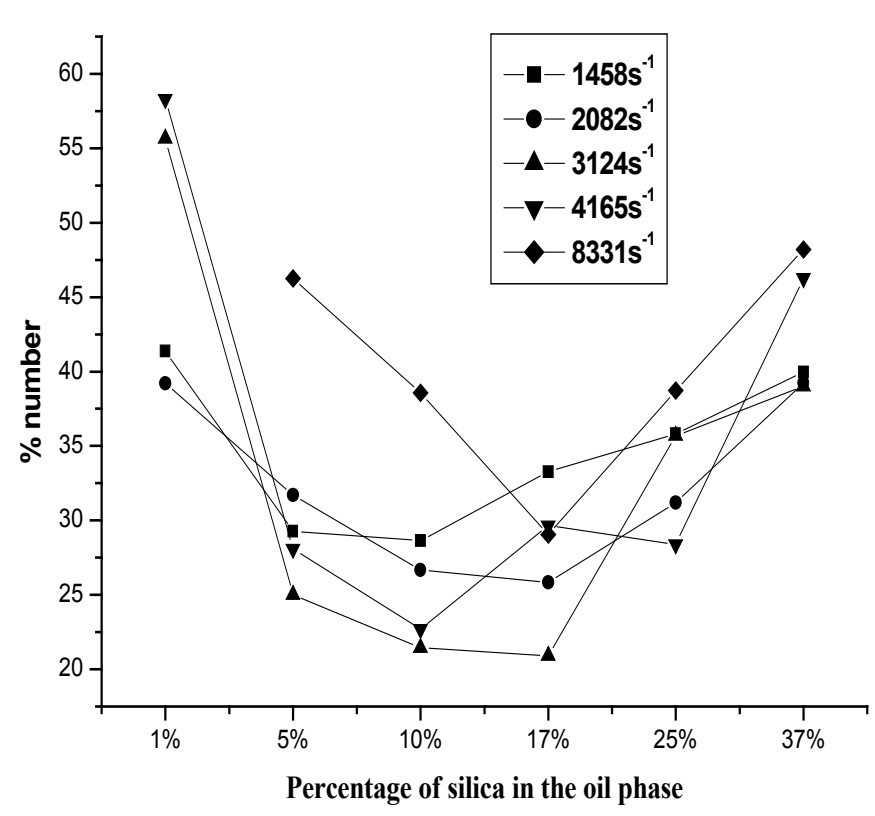

Figure 5 : percentage of single particles in the cluster suspension versus percentage of silica in the oil phase (shear rates : $1458 s^{-1}$ to $8331 s^{-1}$ )

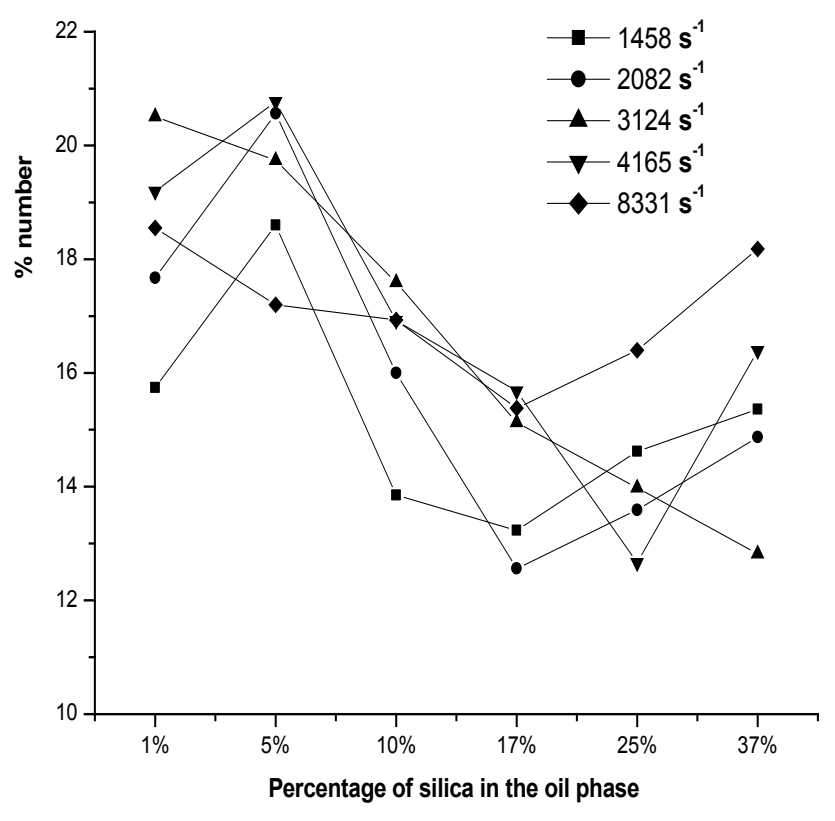

Figure 6 : percentage of doublets in the cluster suspension versus percentage of silica in the oil phase (shear rates : $1458 s^{-1}$ to $8331 s^{-1}$ ) 

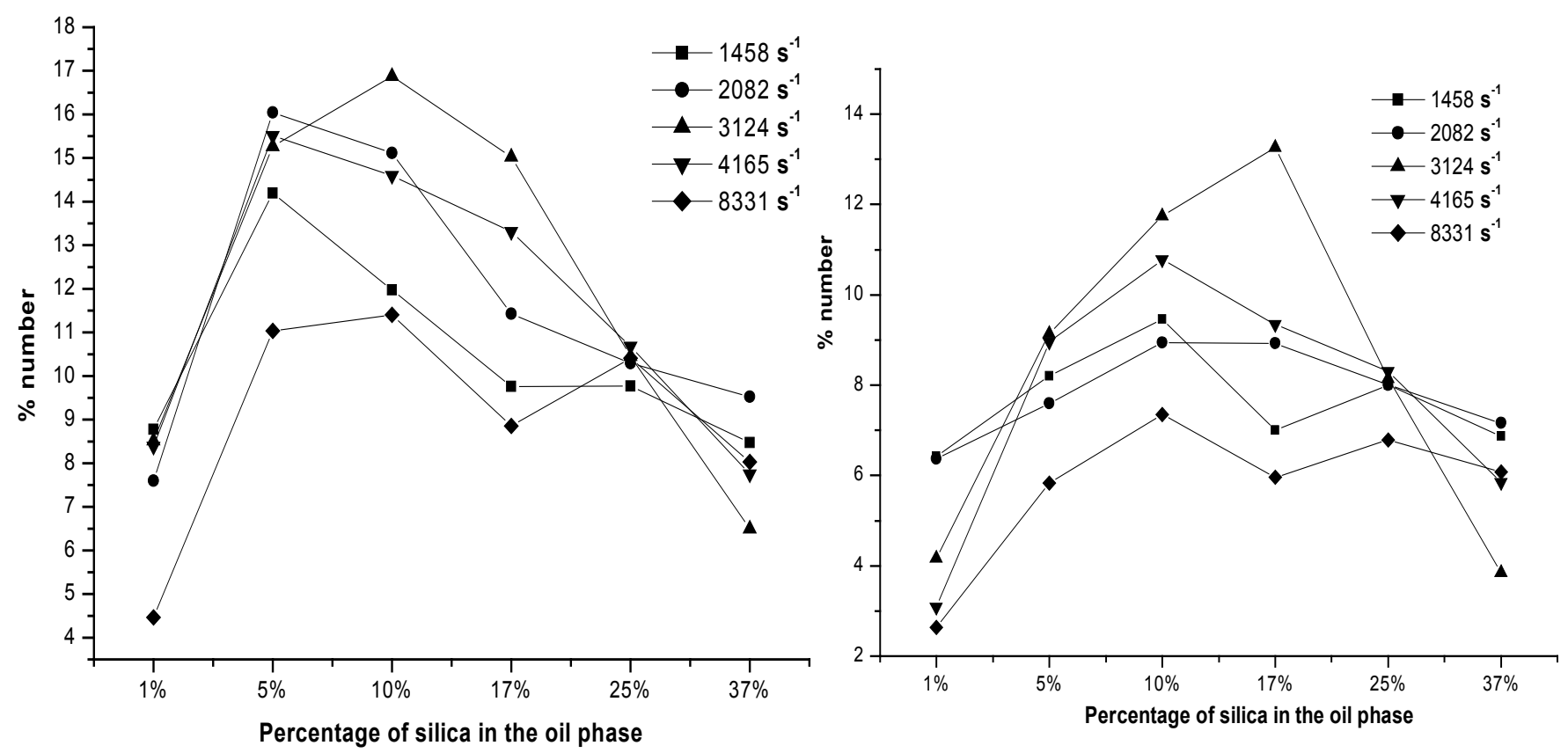

Figure 7 : percentage of triplets in the cluster suspension versus percentage of silica in the oil phase (shear rates : $1458 \mathrm{~s}^{-1}$ to $8331 \mathrm{~s}^{-1}$ )
Figure 8 : percentage of quadruplets in the cluster suspension versus percentage of silica in the oil phase (shear rates : $1458 \mathrm{~s}^{-1}$ to $8331 \mathrm{~s}^{-1}$ )

\section{Conclusion}

In summary, we used the method of emulsion encapsulation originally described by Manoharan et $a l .^{25}$ for making clusters of 2,3 or 4 silica particles with a radius of $1.2 \mu \mathrm{m}$. We showed that careful control of the conditions of emulsification leads to emulsions with a narrow size distribution and leads to suspensions with a high percentage of 2, 3 and 4 aggregates. Our best results were obtained using low to moderate shear rates, a highly viscous continuous phase, and intermediate amounts of silica $\left(\phi_{\text {int }}=5\right.$ to $17 \%$ ) in the droplet phase. Increasing the concentration of silica from $5 \%$ to $17 \%$ favors forming aggregates of an increasing number of particles: doublets, triplets and quadruplets. The separation of the colloidal solution by sedimentation in a concentration gradient led to aqueous suspensions of identical 
clusters. Our preparation process of quasi-monodisperse emulsions can be performed on a large scale and hence, we are capable of making very large amount of crude cluster suspensions with increased yields in small aggregates. The cluster separation remains a limitating step. However our process already produces significantly greater quantities of identical colloidal clusters than previously possible. Since the sedimendation step can certainly be scaled up, it should be possible to produce even larger quantities, so that the thermodynamic and flow properties of these new colloidal clusters can be investigated. These clusters may also serve as building blocks for more complex assemblies, particularly colloidal crystals which might find an application as photonic materials.

Acknowledgements. We thank E. Bertrand and D. Lootens for help and fruitful discussions.

We also thank S. Borensztajn for the scanning electron micrographs. D. J. Pine acknowledges support from the U.S. National Science Foundation (CTS-0221809).

\section{References}

(1) P. N. Pusey, W. Van Megen, P. Barlett, B. J. Ackerson, J. G. Rarity, S. M. Underwood, Phys. Rev. Lett., 1989, 63, 2753

(2) U. Gasser, E. R. Weeks, A. Schofield, P. N. Pusey, D. Weitz, Science, 2001, 292, 258

(3) H. Miguez, F. Meseguer, C. Lopez, A. Mifsud, J. S. Moya, L. Vazquez, Langmuir, 1997, 13, 6009

(4) (a) A. Van Blaaderen, P. Wiltzius, Science, 1995 , 270, 1177.

(b) A. Yetrihaj, A. Van Blaaderen, Nature, 2003, 421, 513.

(5) J. H. Holtz, S. A. Asher, Nature, 2003, 289, 829. 
(6) (a) :R. C. Schroden, C. F. Blanford, B. J. Melde, B. J. S. Johnson, A. Stein, Chem. Mater., 2001, 13,1074

(b) B. J. S. Johnson, A. Stein, Inorg. Chem., 2001, 40, 801

(7) S. I. Matsushita, T. Miwa, D. A. Tryk, A. Fujishima, Langmuir, 1998, 14, 6441

(8) O. D. Velev, E. Kaler, Adv. Mater., 2000, 12, 531

(9) J. G. C. Veinot, H. Yan, S. M. Smith, J. Cui, Q. Huang, T. J. Mark, Nano Lett., 2002, 2, 333

(10) Y. Xia, B. Gates, Z.-Y. Li, Adv. Mater., 2001, 13, 409

(11) D. J. Norris, Y. A. Vlasov, Adv. Mater., 2001, 13, 371

(12) Y. A. Vlasov, X-Z. Bo, J. C. Sturm, D. J. Norris, Nature, 2001,414, 289

(13) W. Wang, B. Gu, L. Liang, W. A. Hamilton, J. Phys. Chem. B, 2003, 107, 12113

(14) Y. Xia, B Gates, Y Yin, Y Lu, Adv. Mater., 2000, 12, 693

(15) Y Lu, Y Yin, Y Xia, Adv. Mater., 2001, 13, 415

(16) A. Van Blaaderen, Science, 2003, 301, 470

(17) J. Azienberg, P. V. Braun, P. Wiltzius, Phys. Rev. Lett., 2000, 84, 2997

(18) K. Chen, X. Jang, L. C. Kimerling, P. T. Hammond, Langmuir, 2000, 16, 7825

(19) (a) Y. Yin, Y. Xia, Adv. Mater., 2001, 13, 267

(b) Y. Yin, Y. Lu, B. Gates, Y. Xia, J. Am. Chem. Soc., 2001, 123, 8718

(20) Y. Xia, Y. Yin, Y. Lu, J. Mc Lellan, Adv. Funct. Mater., 2003, 12, 907

(21) O. D. Velev, K. Furusawa, K. Nagayama, Langmuir, 2000, 16, 2374 
(22) A. D. Dinsmore , M. F. Hsu, M. G. Nikolaides, M. Marquez, A. R. Bausch, D. A. Weitz, Science, $\mathbf{2 0 0 2}, 298,1006$

(23) O. D. Velev, K. Furusawa, K. Nagayama, Langmuir, 2000, 16, 2385

(24) O. D. Velev, A. M. Lenhoff, E. W. Kaler, Science, 2000, 287, 2240

(25) V. N. Manoharan, M. T. Elsesser, D. J Pine, Science 2003, 301,483

(26) G.-R. Yi, V. N. Manoharan, E. Michel, M. T. Elsesser, S.-M. Yang, D. J. Pine, Adv. Mater., $\mathbf{2 0 0 4}, 16,1204$

(27) W. Stöber, A. Fink, J. Colloid Interface Sci., 1968, 26, 62

(28) D. Lootens, Ph.D. Thesis, Université de Paris VI, Paris, France, 2004

(29) J. N. Kinkel, K. K. Unger, J. Chromatography, 1984, 316, 193

(30) C. Goubault, K. Pays, D. Olea, P. Gorria, J. Bibette, V. Schmitt, F. Leal-Calderon, Langmuir, $\mathbf{2 0 0 1}, 17,5184$

(31) T. G. Mason, J. Bibette, Langmuir, 1997, 13, 4600

(32) C. Mabile, V. Schmitt, P. Gorria, F. Leal-Calderon, V. Faye, B. Deminière, J. Bibette, Langmuir, 2000, 16,422

(33) C. Mabile, F. Leal-Calderon, J. Bibette, V. Schmitt, Europhys. Lett., 2003, 61, 708

(34) TSR Co., 88 avenue Victor Hugo, 33700 Merignac, France

(35) L. L. Bondoc and S. Fitzpatrick, Journal of industrial microbiology \& biotechnology 1998, 20, 317 
(36) These values were calculated using the superposition $T$-matrix codes for multi-sphere clusters given in http://www.giss.nasa.gov/ crmim/t_matrix.html. The codes are based on the $T$-matrix method for calculation of the scattering properties of multi-spheres clusters presented in the paper : D. W. Mackowski, M. I. Mishchenko, J. Opt. Soc. Am. A, 1996, 13, 2266.

(37) G. I. Taylor, Proc. R. Soc. A, 1934, 146, 501

(38) H. P. Grace, Chem. Eng. Commun., 1982, 14, 225

(39) For oil-in water emulsions, the ruptured droplets size has been proposed to follow the equation, $\mathrm{R} \approx \mathrm{Cac} \cdot \sigma /\left(\eta_{\mathrm{eff}} \cdot \dot{\gamma}\right)$, where $\mathrm{Cac}$ is the critical capillary number, $\sigma$ is the interfacial tension, $\eta_{\mathrm{eff}}$ is the effective viscosity which is related to the viscosity of the continuous phase and $\dot{\gamma}$ is the applied shear rate.

(40) M. P. Aronson, Langmuir, 1989, 5, 494 


\section{Table of Content Graphic}

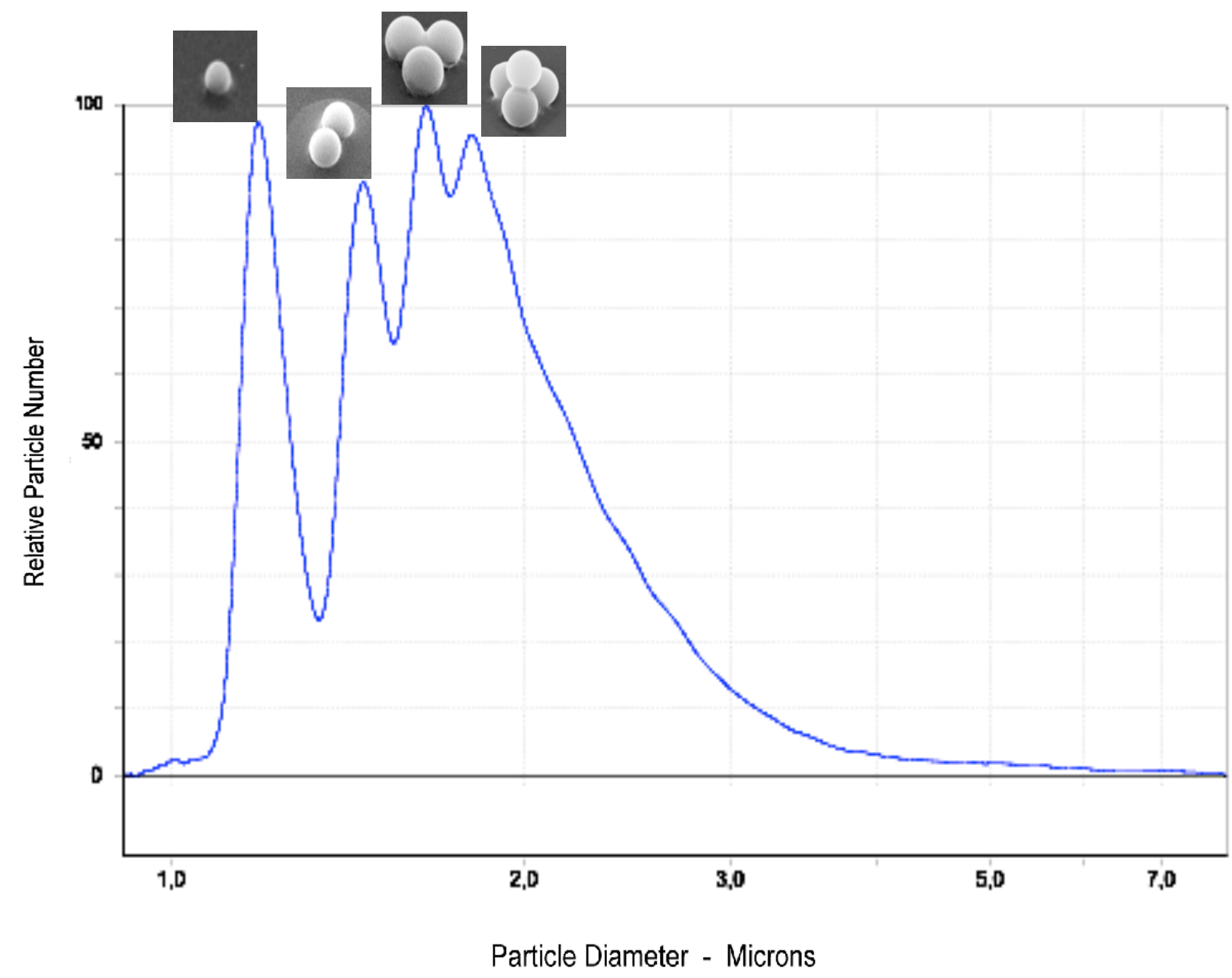

